\title{
Análise da transformada em ondeletas aplicada em sinal geofísico
}

\author{
(Analysis of Wavelet Transform applied in Geophysical) \\ Maurício José Alves Bolzan

\begin{abstract}
Universidade do Vale do Paraíba, Instituto de Pesquisa e Desenvolvimento Laboratório de Física Solar e Atmosférica, Urbanova, São José dos Campos, SP, Brasil.
\end{abstract}

\begin{abstract}
É mostrada uma análise em ondeletas aplicada em um sinal geofísico, número de manchas solares. Para isso, é dado uma introdução teórica sobre a Transformada em Ondeletas e sobre séries e Transformada de Fourier. A Transformada em Ondeletas é uma ferramenta matemática de grande utilidade aplicação em sinais não-estacionários.
\end{abstract}

Palavras-chave: transformada em ondeleta, manchas solares.

\begin{abstract}
A wavelet analysis applied in geophysical signal, sunspot number, it is shown. For this, it is given a theoretical introduction about the wavelets Transform and Fourier Transform and Series. The Wavelet Transform is useful mathematical tool for application in non-stationary signals.
\end{abstract}

Keywords: wavelet transform, sunspot.

\section{Introdução}

Hoje em dia, o estudo de sinais não-estacionários exige abordagens matemáticas robustas de análise para poder compreender os fenômenos físicos que estão agindo em qualquer sistema natural. Uma destas ferramentas é a Transformada em Ondeletas, capaz de analisar séries-temporais obtidas de qualquer sistema físico. A Transformada em Ondeletas tem sido aplicadas nas mais diversas áreas do conhecimento, desde estudos sobre turbulência atmosférica [1], processamento de sinais [2] até sistemas hidrológicos [3]. O uso desta ferramenta se faz necessário devido ao fato de que as séries-temporais tomadas de qualquer sistema físico possuírem características não-estacionárias. Entenda-se como característica não-estacionária as séries-temporais cujo seus momentos estatísticos, média, variância, e etc, variam em qualquer segmentos tomados desta série. Por isso, o objetivo deste trabalho é demonstrar a sua utilidade na análise de qualquer sinal experimental nãoestacionário em tempo e frequência, tomando como exemplo um sinal geofísico, onde poderia ter tomado outro sinal de qualquer sistema físico sem perda nenhuma de generalidade. Este sinal consiste em uma série-temporal do número de manchas solares mensais deste o ano de 1749 até o ano de 2002. A Fig. 1 mostra um gráfico de manchas solares neste período. É fácil ver nesta figura um período predominante de 11 anos, aproximadamente, tendo um máximo em 1958. Até o presente, tem-se 23 ciclos completos.

Antes de iniciar uma introdução da Transformada em Ondeletas faz-se necessário uma breve introdução sobre Séries e Transformada de Fourier. Antes de 1930, Joseph

Enviar correspondência para Maurício José Alves Bolzan. E-mail: bolzan@univap.br.
Fourier, com sua teoria de análise de frequência afirmou que qualquer função periódica $f(x)$ é a somatória de

$$
f(x)=a_{0}+\sum_{\mathrm{k}=1}^{\infty}\left(a_{k} \cos (k x)+b_{k} \operatorname{sen}(k x)\right)
$$

ou seja, qualquer função periódica poderia ser expressa por uma somatória de senos e cossenos. Os coeficientes $a_{0}, a_{\mathrm{k}} \mathrm{e}$ $b_{\mathrm{k}}$ são calculados por

$$
\begin{aligned}
& a_{0}=\frac{2}{\pi} \int_{0}^{2 \pi} f(x) d x, \\
& a_{k}=\frac{1}{\pi} \int_{0}^{2 \pi} f(x) \cos (k x) d x, \\
& b_{k}=\frac{1}{\pi} \int_{0}^{2 \pi} f(x) \operatorname{sen}(k x) d x .
\end{aligned}
$$

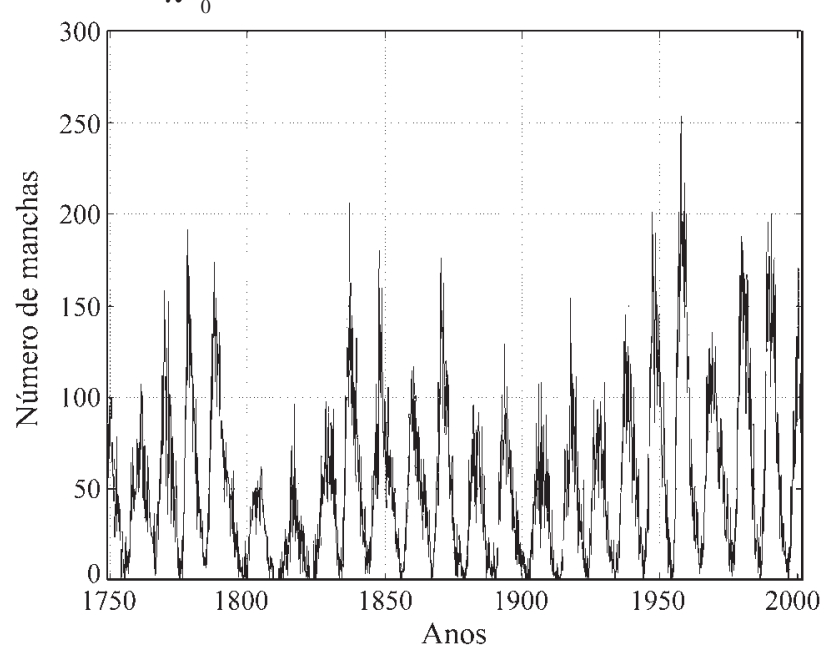

Figura 1 - Série-temporal do número de manchas solares de 1749 até 2002. 
Portanto, representações em série de Fourier de funções como uma superposição de senos e cosenos têm sido muito útil para soluções analíticas e numéricas de equações diferenciais e para análises e tratamento de sinais.

A chamada Transformada de Fourier pode ser considerada como um limite de uma combinação linear infinita de ondas senoidas e que encontra grandes aplicações no tratamento de sinais estacionários. Uma função aperiódica pode ser considerada como o caso limite de uma função periódica cujo período $\mathrm{T} \rightarrow \infty$. Assim, pode-se representar uma série temporal como:

$$
f(t)=\frac{1}{2 \pi} \int_{-\infty}^{+\infty} F(\omega) e^{i \omega t} d \omega,
$$

onde

$$
F(\omega)=\int_{-\infty}^{+\infty} F(t) e^{-i \omega t} d t .
$$

Nas Eqs. 5 e 6 foram utilizadas as funções trigonométricas na forma complexa. Note que, nestas equações ocorre o que chama-se de Convolução. Para esclarecer mais este assunto, faremos uma breve discussão. Sejam $x(t) \mathrm{e}$ $h(t)$ funções reais. Então, por definição, a convolução de $x(t)$ e $h(t)$ é uma função $y(t)$ expressa por

$$
y(t)=x(t) \otimes h(t)=\int_{-\infty}^{+\infty} x(\tau) h(t-\tau) d \tau,
$$

onde $\rightarrow$ representa o processo de convolução. Portanto, $x(t)$ representa uma série temporal qualquer e $h(t)$ representa uma função filtro que tem o papel de identificar e selecionar o período de cada componente oscilatória prensente em $x(t)$.

Os conhecimentos citados acima são utilizados exaustivamente em análise e tratamentos de séries-temporais estacionárias. Portanto, devido a sua deficiente aplicabilidade em séries-temporais não- estacionárias, o que é a maioria dos casos na natureza, a Transformada em Ondeleta foi desenvolvida por Morlet na década de 80 [4]. Meyer [5] demonstrou as condições de ortogonalidade deste novo operador matemático, oferecendo condições seguras para a aplicação da nova técnica.

A idéia central da análise em ondeletas consiste em decompor um sinal a diferentes níveis de resolução, processo conhecido como Multiresolução. A representação de multiresolução fornece uma moldura hierárquica simples para interpretação de informação do sinal. A diferentes resoluções, os detalhes de um sinal geralmente caracterizam diferentes estruturas físicas do mesmo. A uma resolução mais grosseira, estes detalhes geralmente caracterizam as grandes estruturas que fornecem o contexto. Com o aumento da resolução, obtemos detalhes mais finos. $\mathrm{O}$ processo de decomposição ocorre da seguinte maneira. Considere inicialmente uma função $f$ contida no espaço das funções quadraticamente integráveis $L_{2}(R)$. Portanto, a energia da função $f$ é limitada ao longo de todo o eixo dos reais, isto é:

$$
\int_{-\infty}^{+\infty}|f(t)|^{2} d t<\infty .
$$

O produto escalar ou produto interno, denotado pelo símbolo $(\bullet)$, e a norma para o espaço $L_{2}(R)$ são definidos como se segue:

$$
f \cdot g=\int_{-\infty}^{+\infty} f(t) \cdot g(t) d t
$$

e

$$
|f|=\sqrt{f \cdot f}
$$

Se a função $g$ possuir norma unitária, então o resultado do produto escalar entre $f$ e $g$ corresponderá a operação de projeção de $f$ sobre $g$. Considere, agora, um conjunto de funções ortonormais $\Psi_{k}(t), k \in N$, ou seja, ortogonais e de módulo 1 , formando uma base para o espaço $L_{2}(R)$. Pela condição de ortogonalidade:

$$
\Psi_{m} \cdot \Psi_{n}=0
$$

para todo $m$ diferente de $n$.

Definimos então a expansão da função $f$ em uma série ortonormal como sendo a combinação linear das funções base ponderadas pelas projeções de $f$ sobre cada uma delas, isto é,

$$
f(t)=\sum_{k=-\infty}^{\infty}<f, \Psi_{k}>\Psi_{k}(t)
$$

fazendo $F(k)=<f, \Psi_{k}>, k \in N$, e $<f, \Psi_{k}>=f \bullet \Psi_{k}$ é uma outra forma de expressar o produto interno ou escalar.

$$
f(t)=\sum_{k=-\infty}^{\infty} F_{\kappa} \Psi_{k}(t)
$$

onde $F_{k}$, define a transformada da função $f(t)$. Imbutido nesta abordagem, está o fato da base $\Psi_{k}(t)$ possuir suporte igual a toda extensão do eixo real, isto é, cada função base $\Psi_{k}(t)$ decai a zero somente nos limites $\pm \infty$. No entanto, se a base possuir suporte limitado, decaindo para zero muito rapidamente, então a melhor maneira desta base cobrir todo o eixo dos reais será através de translações do tipo:

$\Psi_{k, l}(t)=\Psi_{k}(t-l), l \in N$.

Da mesma maneira, observando a base da Transformada de Fourier dada pela Eq. 5, pode-se constatar que cada função base (as exponenciais complexas) é obtida a partir de simples dilatações na frequência. Reunindo estas duas propriedades, dilatação e translação, em um único protótipo de função base, obtemos as chamadas funções ondeletas, onde as dilatações e as translações são dadas por duas variáveis nomeadas $a$ e $b$, respectivamente. Portanto, o termo ondeleta refere-se a um conjunto de funções com forma de pequenas ondas geradas por dilatações, $\Psi(t) \rightarrow \Psi(2 t)$, e translações, $\Psi(t) \rightarrow \Psi(t+1)$, de uma função base geradora simples $\Psi(t)$, a ondeleta-mãe. Esta deve ser quadraticamente integrável dentro de um intervalo de tempo real ou espaço [L2( $R$ )], isto é, deve apresentar energia finita. A imposição de que a sua 
energia média seja zero, constitui a condição de admissibilidade da função:

$$
\Psi_{a, b}(t)=\frac{1}{\sqrt{2}} \Psi\left(\frac{t-b}{a}\right)
$$

de forma que a expansão em série de ondeletas e a transformada são definidas como

$$
<f, \Psi_{a . b}>=\int_{-\infty}^{+\infty} f(t) \Psi\left(\frac{t-b}{a}\right) d t .
$$

Há dois tipos de funções ondeletas, as Contínuas e as Discretas, cada qual útil para determinadas aplicações.

As ondeletas discretas são utilizadas para a decomposição e filtragem de qualquer série-temporal. A sua aplicabilidade neste aspecto advém do fato de que estas ondeletas não provocam retundâncias de coeficientes entre escalas (frequências) [6]. A ondeleta discreta mais comum é a ondeleta de Haar [7]. A Fig. 2 mostra a ondeleta de Haar, onde nota-se que ela é uma função escada. Como exemplo da aplicabilidade desta função, decompomos a série-temporal do número de manchas solares em todas as escalas (frequências) disponíveis. Por ser discreta, esta categoria de ondeletas trabalham com sinais temporais que tenham comprimentos da ordem de potência de dois mais próxima, ou seja, $2^{n}=s$, onde $s$ é o comprimento total da série, e $n$ é o número de frequências possíveis para a decomposição.

A Fig 3. mostra a série-temporal decomposta nas cinco primeiras frequências do sinal original, enquanto que a Fig. 4 mostra o sinal de baixa frequência do sinal original. Note na Fig. 4 que a decomposição nas últimas seis frequências correspondem aos baixos períodos de oscilação do sinal original. Também, devido a forma da ondeleta de Haar, percebe-se que estas oscilações de baixos períodos tornaram-se retangulares. Este procedimento de decomposição em várias escalas disponíveis através da Transformada em Ondeletas Discretas, permite estudar características particulares de cada frequência, ou seja, estudar fenômenos que ocorrem somente em determinadas escalas (frequências). Além disso, permite realizar filtragens passa-baixo, passa-alto ou passa-banda.

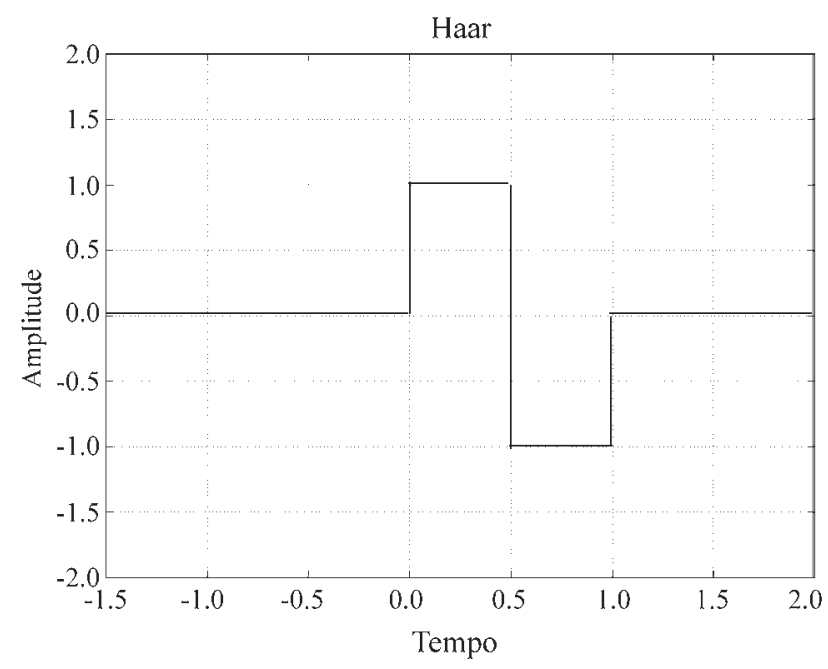

Figura 2 - Função ondeleta de Haar.

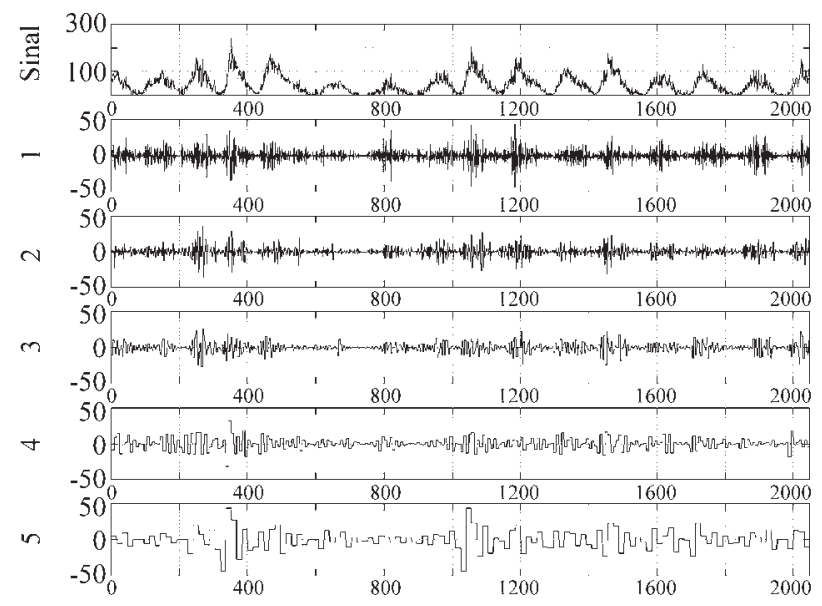

Figura 3 - Decomposição do sinal original (topo) nas cinco primeiras frequências, utilizando a ondeleta de Haar. O eixo horizontal refere-se ao tempo em anos, e o eixo vertical representa a amplitude do sinal em cada frequência.
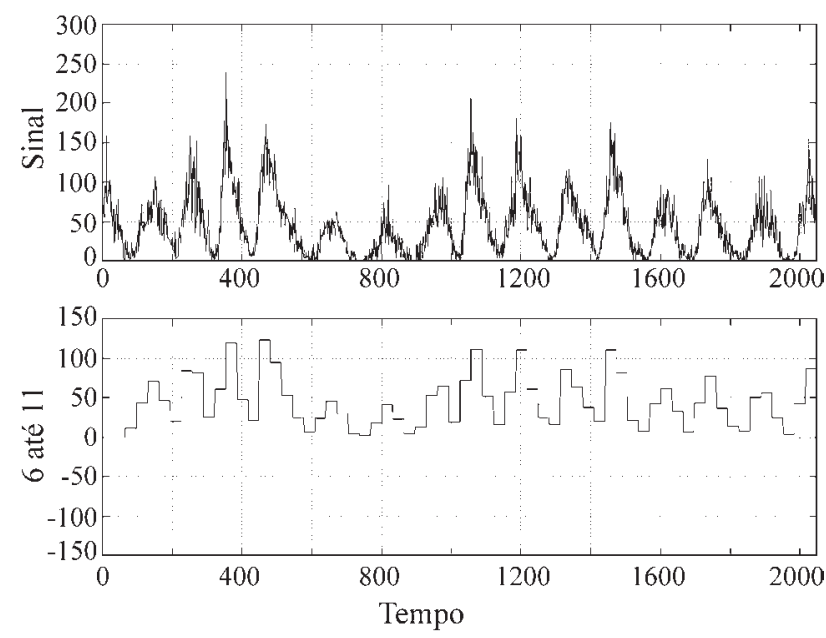

Figura 4 - Decomposição do sinal original (topo), e a soma das seis últimas frequências (abaixo), utilizando a ondeleta de Haar. O eixo horizontal refere-se ao tempo em anos, e o eixo vertical representa a amplitude do sinal.

As ondeletas contínuas são comumentes utilizadas para visualizar, em um diagrama tridimensional, a relação existente entre as componentes de diferentes frequências em função da escala temporal do sinal estudado, onde estas relações são comumentes categorizadas como não-lineares. Fisicamente, em um sistema natural qualquer, é importante tentar buscar relações entre os diversos fenômenos físicos atuantes no sistema natural. Por isso, as ondeletas contínuas propicia um meio matemático adequado para esta busca através do diagrama. Nesta visualização gráfica de uma série-temporal, o eixo y é dedicado a escala de frequências, o eixo x é dedicado a escala de tempo, e por fim, um terceiro eixo dedicado a intensidade de energia (comumente representado por cores em um diagrama). As ondeletas contínuas mais comuns são: a Morlet e a Chapéu Mexicano, dentre outras [7]. A Fig. 5 monstra ambas funções ondeletas contruídas a partir de rotinas disponíveis em Matlab.

Com base na Eq. 16, aplicamos a série temporal das manchas solares em $f(t)$ e a função ondeleta escolhida foi a Morlet. Ainda não há na literatura um consenso de qual a 


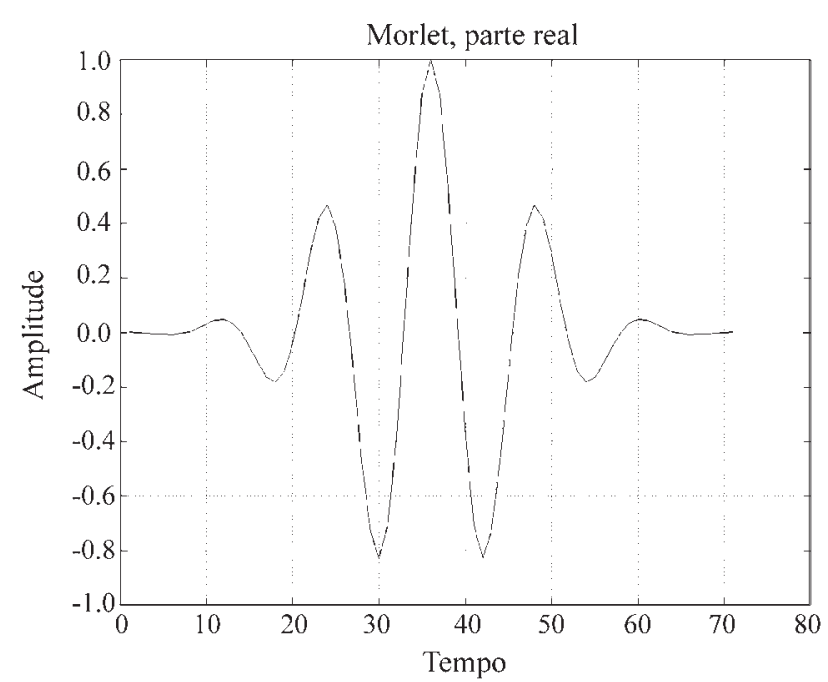

Figura 5 - Funções ondeleta de Morlet e Chapéu Mexicano.

melhor função ondeleta a ser utilizada. O que comumente se aceita é que a função ondeleta a ser utilizada possua um formato característico próximo das características encontradas na série-temporal. A função ondeleta de Morlet é bastante útil para analisar as várias frequências pertencentes ao sistema e mostrar uma relação entre tempo e frequência. A Fig. $6 \mathrm{~b}$ mostra um gráfico onde no eixo vertical é mostrado os períodos em anos encontrados na série

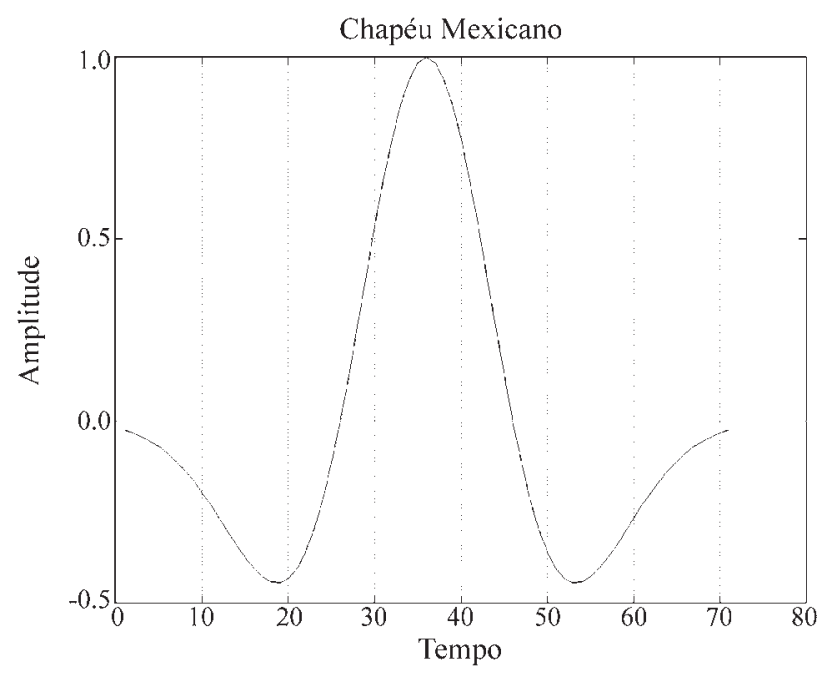

temporal, no eixo horizontal é mostrado o comprimento temporal em anos da série, e os tons de cinza do gráfico representa o módulo da amplitude do sinal em cada escala de frequência, ou seja, representa a energia associada a cada frequência existente no sistema. Note neste gráfico o período dominante de aproximadamente 11 anos, mostrando o ano de 1958 como o ano em que estiveram mais atuantes as manchas solares. É possível perceber também

a) Número de manchas solares

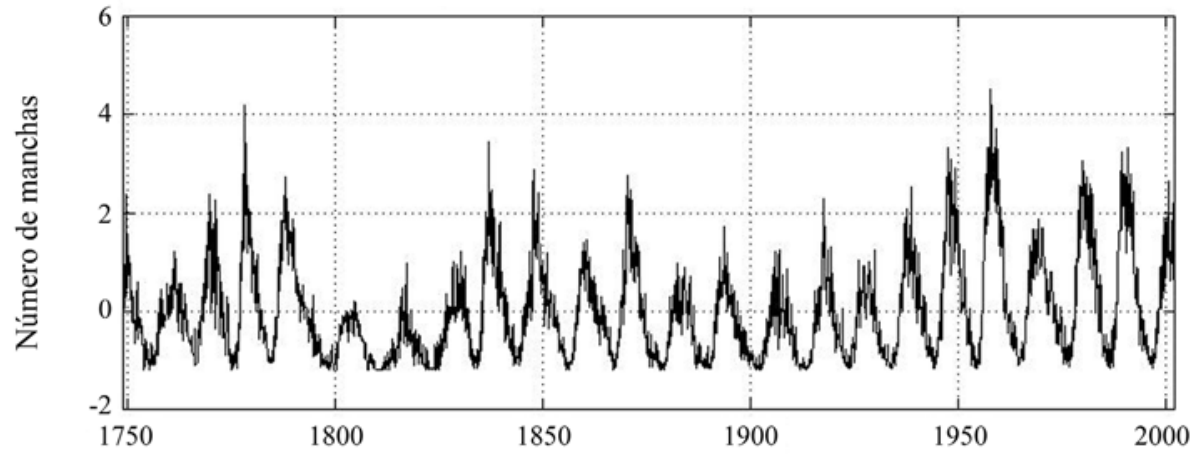

b) Espectro de potência da wavelet para manchas solares

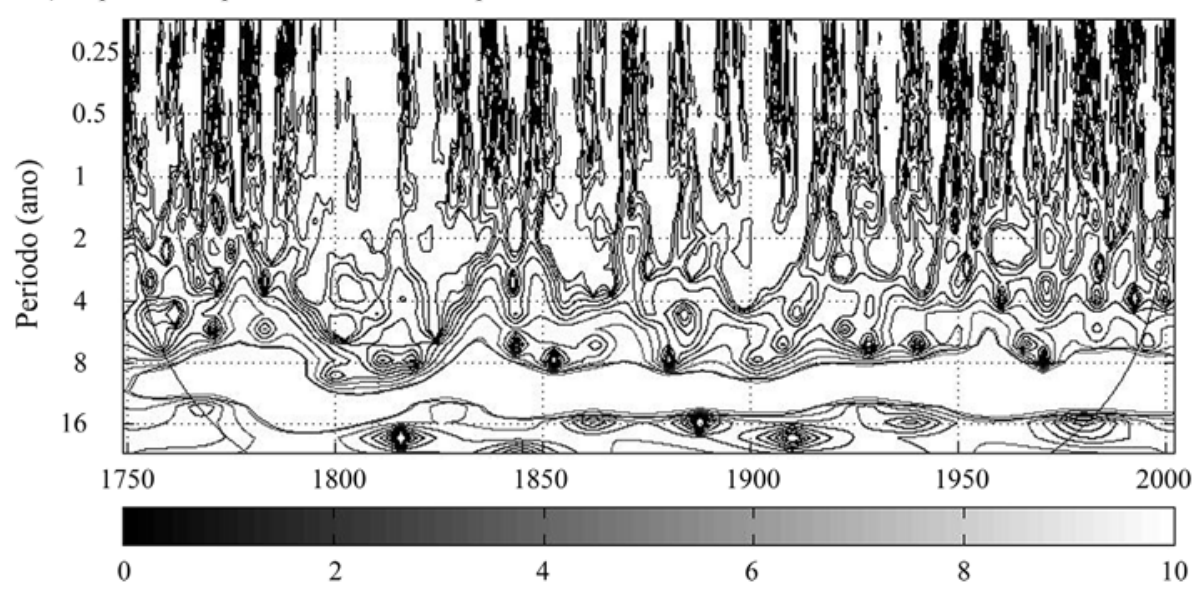

c) Espectro de ondeleta global

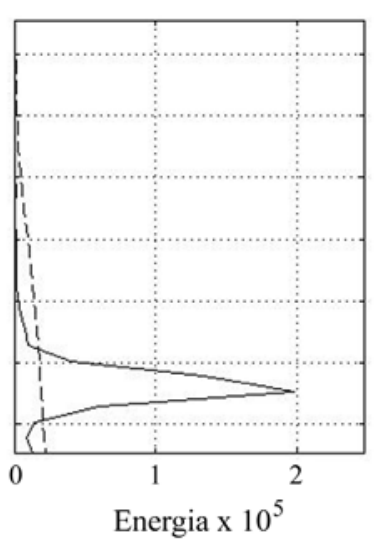

Figura 6 - (a) Valores do número de Manchas Solares por ano, desde 1749 até 2002. (b) Diagrama tempo-frequência da série temporal de manchas solares. (c) Variância (energia) associada a cada período de anos. 
períodos menores que 11 anos, particularmente um período ocorrendo entre 4 a 8 anos.

Com base nestes resultados, foi realizado o cálculo da variância (energia) em cada um destes períodos de anos. $\mathrm{O}$ objetivo disso é saber quais os períodos com maior energia. Para realizar esta tarefa, utilizou-se um procedimento no qual consiste em somar toda a energia associada a cada período ou escala $a$, de acordo com a Eq. 17. Este procedimento, conhecido na literatura científica pelo termo em inglês de Global Wavelet Spectra (Espectro de Ondeleta Global), é uma forma similar do espectro de energia obtido via Transformada Rápida de Fourier, conhecida pela sigla FFT. Na Fig. 6c, o eixo das ordenadas (vertical) refere-se ao período em anos, o mesmo da Fig. 6b, e o eixo da abscissa representa a variância (energia) associada com cada período em anos. Percebe-se nitidamente que o pico de máxima variância está associada justamente com o período de 11 anos, aproximadamente. Além disso, após esse máximo de variância ocorre uma forte queda e aumenta posteriormente, em direção a períodos cada vez maiores, acima de 16 anos, e uma pequena variância ocorrendo até o período de 4 anos. No entanto, não pode-se inferir sobre períodos superiores a 16 anos através desta análise de ondeleta pois, o nível de significância dos resultados fica comprometido, já que ainda não possui uma série-temporal suficientemente longa.

$$
M(a)=\int|W(a, t)|^{2} d t .
$$

Muitos autores têm realizado estudos sobre as periodicidades nestes dados de manchas solares. Alguns pesquisadores em estudos iniciais [8, 9], usaram métodos de periodogramas e harmônicos, outros [10] usaram a análise espectral de Blackman e Tukey [11] para estudar períodos menores do que 11 anos e determinaram periodicidades de 5.5 e 3.5 anos, mas o nível de significância foi inferior a 95 por cento.

Os resultados obtidos aqui, via análise em ondeletas, demonstram consistência com os resultados encontrados na literatura, tornando-se assim uma ferramenta de análise robusta de séries-temporais por causa da sua grande aplicabilidade em sinais não-estacionários. Permitindo com isso, realizar novas abordagens que até então não eram possíveis de serem realizadas com outras ferramentas de análises. Além disso, já se encontra disponível na internet pacotes de ondeletas para vários tipos de linguagens como o Fortran, Matlab, IDL, dentre outros.

\section{Conclusões}

O uso da Transformada em Ondeletas aplicada em um sinal geofísico, número de manchas solares, demonstrou ser uma ferramenta matemática e robusta para análise de sinais com características não-estacionárias. Os resultados obtidos aqui demonstraram consistência com trabalhos de vários pesquisadores que utilizaram outras ferramentas matemáticas de análise. Além disso, a sua aplicabilidade hoje em dia tornou-se acessível devido ao grande número de subrotinas disponíveis em vários softwares, tais como Fortran, Matlab e IDL. Existem muitas páginas na internet dedicadas ao assunto das Ondeletas nas mais diversas aplicações. Porém, há duas páginas que considero muito importantes, são elas: http://paos.colorado.edu/research/ wavelets/ e http://www-stat.stanford.edu/ wavelab/. Portanto, espera-se que este artigo possa ter motivado alunos para a sua aplicação em sinais temporais obtidos de diversos sistemas físicos.

\section{Referências}

[1] C. Rodrigues Neto, A. Zanandrea, F.M. Ramos, R.R. Rosa, M.J.A. Bolzan, and L.D.A. Sá, Physica A 295, 215 (2001).

[2] M. Vertteli, C. Herley, IEEE Trans. Signal Proc. 40, 2207 (1992).

[3] D.J.R. Nordemann, Pesq. Agropec. Bras. 33, 1787 (1998).

[4] M. Farge, Annual Review of Fluid Mechanics 24, 395 (1992).

[5] Y. Meyer, Ondelettes - Ondelettes et Opérateurs I (Cambridge University Press, Cambridge, 1990).

[6] I. Daubechies, Ten Lectures on Wavelets (Society for Industrial and Applied Mathematics, Philadelphia, PA, 1992).

[7] P.A. Morettin, Ondas e Ondeletas (EdUSP, São Paulo, 1999).

[8] H. Kimura, Monthly Notices R. Astron. Soc. 73, 543 (1913).

[9] H.H. Turner, Monthly Notices R. Astron. Soc. 73, 714 (1913).

[10] Rao K. Ramanuja, Solar Physics, 29, 47 (1973).

[11] R.B. Blackman and J.W. Tukey, The Measurement of Power Spectra (Dover, New York, 1959). 